\title{
Análise da linha de cuidado para pacientes com diabetes mellitus e hipertensão arterial: a experiência de um município de pequeno porte no Brasil
}

\author{
Monica Viegas Andrade* \\ Kenya Noronha** \\ Cláudia Di Lorenzo Oliveira ${ }^{\star \star \star}$ \\ Clareci Silva Cardoso ${ }^{\star \star \star \star}$ \\ Júlia Almeida Calazans ${ }^{\star \star \star \star \star}$ \\ Nayara Abreu Julião $0^{\star \star \star \star \star \star}$ \\ Aline de Souza ${ }^{\star \star \star \star \star \star \star}$ \\ Patrícia Aparecida Tavares
}

\begin{abstract}
0 artigo avalia a linha de cuidado de uma coorte de 260 indivíduos com diabetes mellitus e 295 indivíduos com hipertensão arterial sistêmica antes, durante e após a implantação do Laboratório de Inovações na Atenção às Condições Crônicas (LIACC). Essa intervenção buscou fortalecer a atenção primária à saúde, implantando o modelo de atenção às condições crônicas no município de Santo Antônio do Monte, Minas Gerais, Brasil, entre 2013 e 2014. Trata-se de um estudo observacional longitudinal que utiliza informações clínicas e laboratoriais dos prontuários dos pacientes dessas duas condições crônicas entre 2012 e 2017. Os desfechos avaliados foram baseados nas linhas guias da Secretaria de Estado de Saúde de Minas Gerais. Os resultados evidenciam o LIACC associado à universalização de macroprocessos da atenção primária, como o cadastramento e a classificação do risco familiar. Para pacientes com diabetes houve melhora em diversos marcadores no período, como o aumento da realização de consulta (de $90 \%$ em 2012 para 92\% em 2017) e diminuição dos pacientes com exames fora das faixas de normalidade. Já para indivíduos com hipertensão, mesmo sendo observado um crescimento acentuado da realização de consultas (de 80\% em 2012 para 84\% em 2017), as melhorias clínicas foram menos evidentes. Conclui-se que o LIACC se configura como uma promissora intervenção para a melhoria do manejo de pacientes com doenças crônicas na atenção primária à saúde.
\end{abstract}

Palavras-chave: Condições crônicas. Atenção primária à saúde. Estudos de coorte.

\footnotetext{
* Departamento de Economia, Centro de Desenvolvimento e Planejamento Regional (Cedeplar), Universidade Federal de Minas Gerais (UFMG), Belo Horizonte-MG, Brasil (mviegas@cedeplar.ufmg.br; https://orcid.org/0000-0002-6821-1598).

${ }^{\star *}$ Departamento de Economia, Centro de Desenvolvimento e Planejamento Regional (Cedeplar), Universidade Federal de Minas Gerais (UFMG), Belo Horizonte-MG, Brasil (knoronha@cedeplar.ufmg.br; https://orcid.org/0000-0002-7174-6710).

*** Departamento de Saúde Coletiva, Universidade Federal de São João del-Rei, campus Centro-Oeste, Divinópolis-MG, Brasil (dlorenzo@ufsj.edu.br; https://orcid.org/0000-0001-8533-8155).

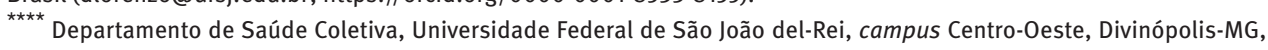
Brasil (clareci@ufsj.edu.br; https://orcid.org/0000-0003-0689-1644).

${ }_{* \star \star \star \star}$ Departamento de Demografia, Centro de Desenvolvimento e Planejamento Regional (Cedeplar), Universidade Federal de Minas Gerais (UFMG), Belo Horizonte-MG, Brasil (jucalazans@cedeplar.ufmg.br; https://orcid.org/0000-0002-6215-3251).

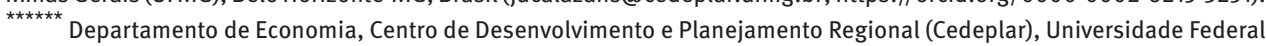
de Minas Gerais (UFMG), Belo Horizonte-MG, Brasil (najuliao@cedeplar.ufmg.br; https://orcid.org/0000-0001-8860-9643).

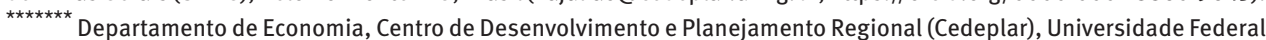
de Minas Gerais (UFMG), Belo Horizonte-MG, Brasil (asouza@cedeplar.ufmg.br; https://orcid.org/0000-0002-3223-9468).

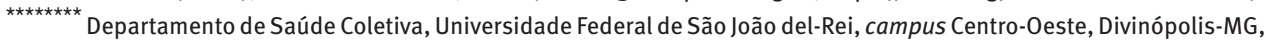
Brasil (patricia.tavares@prof.una.br; https://orcid.org/0000-0002-3626-5217).
} 


\section{Introdução}

Ao longo das últimas décadas, o Brasil vem passando por um rápido processo de transição epidemiológica, caracterizado pelo aumento da carga de condições crônico-degenerativas. Entre estas se destacam a hipertensão arterial sistêmica e o diabetes mellitus, cujo avanço vem gerando uma pressão sobre os custos dos sistemas de saúde e a necessidade de estratégias preventivas voltadas ao controle dessas doenças (MALTA et al., 2017; BAHIA et al., 2011; DE ALMEIDA-PITITTO et al., 2015; LOBO et al., 2017; POBLETE et al., 2018). Segundo dados do suplemento de saúde da Pesquisa Nacional por Amostra de Domicílios (PNAD) de 1998 e da Pesquisa Nacional de Saúde (PNS) de 2013, a prevalência de diabetes no Brasil aumentou de 2,79\% para 7,05\% e a prevalência de hipertensão passou de $15,04 \%$ para $22,06 \%$, entre 1998 e 2013 .

Um dos principais responsáveis pelo aumento da prevalência da hipertensão e do diabetes é o envelhecimento populacional (COELI et al., 2002; SARTORELLI; FRANCO, 2003; ROSA et al., 2007). Contudo, diversos estudos, tanto para o Brasil como para outros países da América Latina e Caribe, mostram que a prevalência de doenças crônicas vem crescendo também nos grupos etários mais jovens (SARTORELLI; FRANCO, 2003; SCHMIDT et al., 2011; CANUDAS ROMO et al., 2014). Dessa forma, esse processo é decorrente da interação da transição demográfica com diversos outros fatores, tais como determinantes hereditários, predisposição genética e condições socioeconômicas e comportamentais. A redução da prática de atividades físicas, o consumo excessivo de bebidas alcoólicas e o aumento da ingestão de alimentos com alto valor calórico, como açúcar e gorduras saturadas, e sódio levam a maiores riscos cardiovasculares e metabólicos glicêmicos, sendo, por isso, frequentemente apontados na literatura como determinantes comportamentais do aumento das condições crônicas (POPKIN et al., 2012; CONDE; MONTEIRO, 2014; HORIUCHI, 1999; BELTRÁN-SÁNCHEZ et al., 2013; CANUDAS ROMO et al., 2014).

É importante destacar também que as regiões brasileiras estão em diferentes estágios nos processos de transição demográfica e transição epidemiológica. Por consequência, podem-se observar importantes diferenciais regionais na carga de diabetes e hipertensão no contexto brasileiro, com as regiões mais pobres apresentando as maiores taxas de prevalência e de mortalidade por essas duas condições (BRASIL, 2011). Esses diferenciais são agravados pela maior dificuldade de acesso a serviços de saúde por parte da população mais pobre e pela pior qualidade de manejo clínico, em termos de diagnóstico, tratamento continuado e atendimento das crises agudas (KLAFKE et al., 2014).

Parte dos agravos relacionados a condições crônicas pode ser contornada e até mesmo evitada por mudanças comportamentais, com a adoção de hábitos de saúde saudáveis, ou pela adoção de estratégias no âmbito da atenção primária sobre o manejo clínico dessas doenças. De forma geral, a atenção primária, especialmente a saúde da família, assume um papel fundamental neste processo, fazendo o levantamento epidemiológico e propondo medidas preventivas, de controle e tratamento (MALFATTI; ASSUNÇÃO, 2011). 
Apesar dos avanços observados no Brasil no sistema público de saúde nos últimos anos, principalmente em relação à cobertura da atenção primária, a organização atual da oferta desses serviços permanece fragmentada e com pouca interação entre os diferentes níveis de atenção. Essa organização do cuidado é capaz de resolver problemas ou complicações relacionados a eventos agudos, entretanto, é pouco eficaz para o cuidado das doenças crônicas que necessitam de um acompanhamento contínuo, proativo e integrado. Algumas iniciativas têm sido implementadas pelo Ministério da Saúde de forma a tornar a organização dos serviços ofertados pelo Sistema Único de Saúde (SUS) coerente com a demanda por cuidado imposta por esse novo perfil epidemiológico. Dentre essas iniciativas, destacam-se as Redes de Atenção à Saúde para o Cuidado Crônico (RAS-CC). Esses mecanismos são arranjos organizacionais que visam atender às demandas de saúde decorrentes da transição epidemiológica e demográfica, na medida em que buscam garantir a integralidade e continuidade do cuidado às condições crônicas (BRASIL, 2010; MALTA; MERHY, 2010). No SUS, as RAS constituem um novo modelo de cuidado que busca superar os vazios assistenciais e reorganizar os processos na atenção primária à saúde (APS) (BRASIL, 2010).

As linhas de cuidados são a principal estratégia para a organização e implantação das RAS, cuja construção deve incluir a articulação entre processos institucionais (VENÂNCIO; ROSA; BERSUSA, 2016; MENDES, 2012). Do ponto de vista dos macroprocessos da atenção primária (cadastramento dos usuários, classificação de risco familiar, estratificação de risco individual, entre outros), a efetivação da linha de cuidado depende das ações de organização da vigilância, informação e comunicação em saúde, mudanças na legislação, organização da rede de serviços e identificação de grupos de risco e elaboração de protocolos. Na perspectiva dos microprocessos da atenção primária (práticas de higienização, controle de estoque, segurança do paciente, assistência farmacêutica, entre outros), é fundamental a atuação da equipe na coordenação do cuidado, vinculação e responsabilização do cuidador e busca da produção da autonomia do usuário (VENÂNCIO; ROSA; BERSUSA, 2016).

Em Minas Gerais, o processo de implementação das RAS-CC e a organização das APS se iniciaram em 2003, com a proposta de substituição do modelo até então vigente por um modelo de saúde integrado e orientado para atender às demandas das condições crônicas com ações proativas, baseadas no cuidado familiar. As iniciativas implementadas no estado ocorreram antes da portaria publicada pelo Ministério da Saúde para a estruturação das RAS (DE CASTRO MOREIRA et al., 2012). Apesar de tentativas para o seu fortalecimento pelos governos estadual e federal, o cuidado ainda é ofertado de forma fragmentada e hierarquizada (FERTONANI et al., 2015).

Considerando essa perspectiva, o Laboratório de Inovação à Atenção às Condições Crônicas (LIACC) propõe um novo modelo de manejo baseado no Modelo de Atenção às Condições Crônicas (MACC) desenvolvido por Mendes (MALTA; MERHY, 2010; MENDES, 2012). Nesse modelo, o conceito de condições crônicas não se restringe à presença de 
doenças crônicas, englobando também qualquer condição de saúde que persista ao longo do tempo e requeira gerenciamento contínuo (MALTA; MERHY, 2010; COSTA et al., 2017). 0 monitoramento adequado dessas condições demanda a criação de uma rede que inclui serviços de saúde primários, secundários e terciários ofertados de forma integral e longitudinal.

No Brasil, o LIACC foi implementado como projeto-piloto em três cidades: Curitiba (2011) no Paraná; Santo Antônio do Monte (2013) em Minas Gerais; e Tauá (2014) no Ceará. Em Santo Antônio do Monte (SAMONTE), a intervenção ocorreu entre junho de 2013 e dezembro de 2014, focando quatro grupos-alvo: diabetes mellitus (DM); hipertensão arterial sistêmica (HAS); gestantes; e crianças menores de dois anos. Os novos processos de organização do cuidado foram introduzidos por meio de oficinas com os profissionais de saúde. Os macroprocessos efetivamente implementados foram o (re)cadastramento das famílias, territorialização, classificação de risco familiar, estratificação de risco individual, implantação do sistema de triagem de Manchester nas UBS, organização da agenda e programação da marcação de consultas, elaboração do plano de cuidado e autocuidado apoiado.

O objetivo do presente estudo é avaliar a linha de cuidado de uma coorte de indivíduos com diabetes e hipertensão na rede de serviços de saúde de SAMONTE antes, durante e após a implantação do LIACC, a partir das informações dos prontuários clínicos dos indivíduos na APS. No Brasil são escassos os trabalhos que avaliam a implementação das linhas de cuidado. Até o momento, foi localizado somente um artigo que realiza uma avaliação da implementação dos macros e microprocessos a partir de entrevistas com gestores, médicos e usuários (MENDES, 2011). Nesse sentido, este artigo traz uma contribuição inédita ao acompanhar longitudinalmente os indivíduos sob a intervenção.

\section{Metodologia}

\section{Fonte de dados}

Trata-se de um estudo descritivo da linha de cuidado de uma coorte de indivíduos com diabetes e hipertensão antes (2012), durante (2013 e 2014) e após (2015, 2016 e 2017) a implantação do LIACC em SAMONTE. Para a construção da linha de cuidado, foram utilizadas informações dos prontuários clínicos da APS em uma amostra representativa de indivíduos identificados por meio de dois inquéritos domiciliares conduzidos no município em 2013 e 2015.

Em 2013, a partir uma pesquisa de base populacional realizada no município, estimaram-se taxas de prevalência de diabetes $(4,53 \%)$ e hipertensão $(26,55 \%)$ em adultos. Com base nessas prevalências, foi produzido um inquérito domiciliar com uma amostra representativa para cada uma dessas condições. Esse inquérito domiciliar de 2013 foi a pesquisa principal para identificação das pessoas que teriam seu prontuário investigado, sendo entrevistados 308 e 364 indivíduos, acima de 18 anos, que se autodeclararam, respectivamente, com diabetes e hipertensão. Quando uma pessoa apresentava as duas 
condições, ela era investigada na amostra de indivíduos com diabetes, uma vez que tal condição registra menor prevalência da população brasileira.

As informações dos prontuários clínicos foram coletadas em dois momentos: maio/junho de 2015 e junho/julho de 2018. Entre as duas coletas de prontuário, é possível ocorrer atrição na base de dados (óbito e migração). A fim de minimizar possíveis problemas de composição da amostra de prontuários clínicos e assim garantir a representatividade, no período estudado, da população com diabetes e hipertensão do município, foi necessário repor os indivíduos que tinham prontuário na UBS em 2015, mas que não foram encontrados em 2018. Essa reposição levou em consideração o grupo etário e o sexo. Os indivíduos incluídos na reposição foram identificados em um inquérito domiciliar em 2015 (VIEIRA et al., 2016).

Foram encontrados, após a inclusão dos prontuários de reposição, 260 indivíduos com diabetes e 295 indivíduos com hipertensão, representando 84,4\% e 81\% da amostra, respectivamente. Os resultados do modelo logístico, estimado para avaliar a presença de seletividade dos indivíduos cujo prontuário foi encontrado, apontam para a ausência de seletividade, uma vez que nenhuma característica socioeconômica ou demográfica foi estatisticamente significativa a nível de $5 \%$ para explicar a chance de o prontuário ter sido localizado.

Para a coleta de dados de prontuário foi utilizado o software Questionnaire Design System ${ }^{\circledR}$. Esta pesquisa foi aprovada pelo Comitê de Ética em Pesquisa da Universidade Federal de São João del-Rei (Processo CEP 369.942).

\section{Indicadores e variáveis}

Foram analisados marcadores clínicos utilizados pela APS para o acompanhamento dos indivíduos com diabetes e hipertensão preconizados pelas Linhas Guias da Secretaria de Estado de Saúde de Minas Gerais (SES/MG) (ANDRADE et al., 2017, 2019). 0 primeiro grupo de indicadores refere-se aos macroprocessos básicos da APS: estratificação de risco individual e classificação de risco familiar. A classificação de risco familiar considera condições de vulnerabilidade socioeconômica das famílias, presença de idosos de alto risco ou frágeis, gestantes, crianças e adolescentes de alto risco e presença de patologias ou condições crônicas prioritárias. Essas informações são obtidas pelos agentes comunitários de saúde a partir de visitas domiciliares. A estratificação de risco individual baseia-se na condição de saúde dos indivíduos com condições crônicas. Para pessoas com diabetes, a estratificação é realizada de acordo com a dosagem de glicohemoglobina, condição de autocuidado do paciente, presença de internação por condições agudas nos últimos 12 meses e complicações crônicas. Para aquelas com hipertensão, além da capacidade de autocuidado, a estratificação considera o risco de um evento cardiovascular, condições clínicas associadas e presença de lesões em órgãos-alvo. 
O segundo grupo refere-se à utilização de serviços ofertados diretamente nas UBS. Para cada ano foram avaliadas consultas médicas ou com outros profissionais de saúde e a realização de exames. 0 perfil lipídico foi analisado para todos os indivíduos. Para aqueles com hipertensão, foi avaliada ainda a medição da pressão arterial (PA) e, para os com diabetes, foi investigada a realização de exames de controle glicêmico (glicohemoglobina e glicemia de jejum). Para cada ano foi construída uma medida binária, indicando a presença do registro no prontuário do exame avaliado. Adicionalmente, elaborou-se um indicador que informa o número de anos em que cada paciente realizou esses exames durante o período investigado. Também coletaram-se informações sobre os seus respectivos resultados, que foram analisadas a partir dos parâmetros estabelecidos pelas Linhas Guias da Secretaria de Estado de Saúde de Minas Gerais (ANDRADE et al., 2017, 2019) definidos como:

- colesterol total (mg/dL): «160 ótimo; 160-199 desejável; 200-239 limítrofe; 240-279 alto; $\geq 280$ muito alto;

- LDL (mg/dL): <100 desejável; $\geq 100$ alto;

- triglicérides (mg/dL): < 150 desejável; $\geq 150$ alto;

- pressão arterial diastólica ( $\mathrm{mmHg}$ ): < 90 risco cardiovascular baixo; $\geq 90$ risco cardiovascular alto. A pressão arterial diastólica vem sendo tradicionalmente utilizada em ensaios clínicos em detrimento da pressão sistólica, uma vez que ela está mais associada a lesões de órgãos (SAFAR, 1988; LUNA; LUNA, 2007);

- glicemia jejum (mg/dL): < 100 controle glicêmico bom; 100-125 controle glicêmico regular; $\geq 126$ controle glicêmico ruim;

- glicohemoglobina: < 7 controle glicêmico bom; 7 a 9 controle glicêmico regular; > 9 controle glicêmico ruim.

Em geral, a avaliação do controle lipídico é realizada com base nos resultados dos exames de colesterol total, fração LDL e HDL e triglicérides. A relação entre esses quatro indicadores permite estimar o resultado de um exame a partir da combinação dos outros três, utilizando-se a equação de Friedewald, a qual fornece estimações precisas quando os triglicerídeos são inferiores a $400 \mathrm{mg} / \mathrm{dL}$, sendo amplamente empregada para estimar o LDL-colesterol sem utilizar ultracentrifugação (PRUITT, 2002). Nesse artigo, usamos essa equação para imputar valores de exames que não estavam registrados no prontuário para os indivíduos com informações em pelo menos três dos exames mencionados. Por último, para os casos em que o paciente realizou o mesmo exame mais de uma vez em um ano, foi considerado o último registro no prontuário.

Além dos indicadores obtidos nos prontuários, foram utilizadas variáveis socioeconômicas, demográficas e de saúde, coletadas durante o inquérito populacional: sexo, idade, UBS de referência, escolaridade, estado de saúde autorreportado, internação nos últimos 12 meses e presença de plano de saúde. 


\section{Método de análise}

0 primeiro passo foi verificar a presença de seletividade devido à perda de indivíduos da amostra cujo prontuário não foi encontrado. Estimou-se um modelo de regressão logística, controlando pelas características socioeconômicas, demográficas e de saúde. A variável dependente assume valor igual a 1 , se o indivíduo teve seu prontuário localizado, e zero, caso contrário.

As mudanças na linha de cuidado implementadas pelo LIACC foram avaliadas a partir de uma análise descritiva dos marcadores selecionados nos prontuários - classificação de risco familiar, estratificação de risco individual e exames. Calcularam-se, para os indicadores contínuos, as medidas de tendência central (média, mediana e desvio padrão) e, para as variáveis categóricas, as proporções. 0 teste de Kruskal-Wallis foi realizado para avaliar a significância das variações entre os anos. A análise foi realizada utilizando o software Stata $13 \AA$.

\section{Resultados}

O Gráfico 1 apresenta os resultados básicos da APS. Aproximadamente 85\% (diabetes) e 95\% (hipertensão) dos prontuários localizados apresentavam a classificação de risco familiar. Na Tabela 1, é possível notar que a maior parte dessa classificação ocorreu nos anos de implementação do LIACC, em 2013 e 2014. Em relação à estratificação de risco individual, houve uma melhora importante entre as duas coletas de dados dos prontuários. Na primeira, em 2015 , somente para $35 \%$ dos indivíduos com diabetes e $28 \%$ daquelas com hipertensão constava o registro de estratificação de risco, enquanto em 2017 tais percentuais se elevaram para 48,9\% e 55,2\%, respectivamente. Esse aumento é importante e ocorreu após a intervenção do LIACC, sugerindo um avanço na implementação desses processos no município. No entanto, a presença de uma parcela considerável de indivíduos sem estratificação individual mostra que esse processo ainda não está consolidado.

As Tabelas 2 e 3 apresentam as informações referentes à realização de consultas e exames por parte dos indivíduos com diabetes e hipertensão, respectivamente. 0 comportamento dos indicadores analisados difere entre os dois grupos. No caso das pessoas com diabetes, observa-se uma melhora tanto na realização dos exames como nos resultados. Isso ocorre logo após a implementação do LIACC e se mantém praticamente constante até o final do período estudado. A proporção de indivíduos que realizaram pelo menos uma consulta na UBS de referência aumentou entre 2012 e 2013, permanecendo relativamente estável nos demais anos. As diferenças entre os anos são estatisticamente significativas a um nível de $5 \%$. Uma análise geral aponta para uma proporção igual ou superior a $90 \%$ ao longo de todo o período analisado. 
GRÁFICO 1

Registros dos indivíduos com diabetes mellitus (DM) e hipertensão arterial sistêmica (HAS), segundo presença de classificação e estratificação de risco

Município de Santo Antônio do Monte, MG - 2012/2017

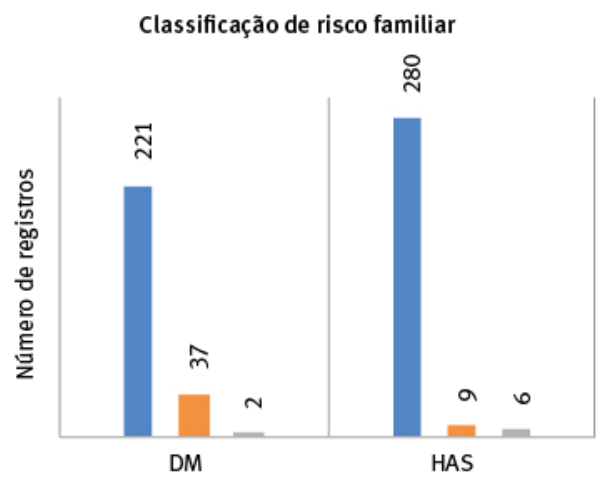

Sim
Estratificação de risco individual

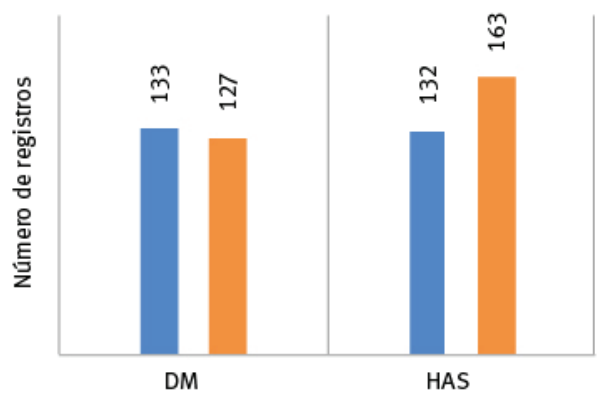

Não sabe

Fonte: SAMONTE $(2013,2015,2018)$.

TABELA 1

Distribuição dos registros de classificação de risco familiar para os indivíduos com diabetes mellitus (DM) e hipertensão arterial sistêmica (HAS) Município de Santo Antônio do Monte, MG - 2009-2017

\begin{tabular}{|c|c|c|c|c|}
\hline \multirow{2}{*}{ Anos } & \multicolumn{2}{|c|}{ DM } & \multicolumn{2}{|c|}{ HAS } \\
\hline & $\mathrm{N}$ & $\%$ & $\mathrm{~N}$ & $\%$ \\
\hline 2009 & 7 & 3,2 & 6 & 2,1 \\
\hline 2010 & 2 & 0,9 & 2 & 0,7 \\
\hline 2011 & 0 & 0,0 & 2 & 0,7 \\
\hline 2012 & 2 & 0,9 & 2 & 0,7 \\
\hline 2013 & 66 & 29,9 & 50 & 17,9 \\
\hline 2014 & 41 & 18,6 & 61 & 21,8 \\
\hline 2015 & 6 & 2,7 & 12 & 4,3 \\
\hline 2016 & 0 & 0,0 & 1 & 0,4 \\
\hline 2017 & 2 & 0,9 & 7 & 2,5 \\
\hline Ano não registrado & 95 & 43,0 & 137 & 48,9 \\
\hline Total & 221 & 100,0 & 280 & 100,0 \\
\hline
\end{tabular}

Fonte: SAMONTE $(2013,2015,2018)$.

Quanto aos exames laboratoriais, de forma geral, para os indivíduos com diabetes que realizaram pelo menos uma consulta na UBS, foram observadas melhoras clínicas importantes. Verifica-se um aumento no registro de exames para o controle lipídico (colesterol total, LDL e triglicérides) ao longo do tempo, sendo as diferenças entre os anos estatisticamente significativas a um nível de $5 \%$. A proporção de indivíduos com o colesterol classificado como ótimo cresceu, entre 2012 e 2017, com simultânea diminuição daquela referente ao colesterol indesejável. No caso do triglicérides, embora verifique-se uma melhora na 
proporção de indivíduos entre os níveis desejáveis, permanece elevada também a proporção de pessoas na faixa considerada alta. Com relação ao controle glicêmico, chama a atenção o aumento expressivo na proporção de indivíduos que realizaram o exame de glicohemoglobina. Enquanto, em 2012, 29\% dos indivíduos com DM tinham registro de realização desse exame no prontuário, em 2017 , essa proporção saltou para $42 \%$. Não obstante, a proporção de indivíduos que realizaram os exames de glicemia e/ou glicohemoglobina permanece abaixo dos $50 \%$ durante todo o período analisado.

Entre as pessoas com hipertensão, os resultados são menos promissores. Apesar de se observar um aumento estatisticamente significativo a um nível de $5 \%$ na proporção de indivíduos que realizaram pelo menos uma consulta na UBS de referência ao longo do período estudado (de 81\% em 2012 para 85\% em 2017), para os exames clínicos e laboratoriais não foram verificadas mudanças importantes depois da implementação do LIACC, sendo que para alguns indicadores houve uma piora nos registros dos prontuários. Em 2012, 80\% dos indivíduos que realizaram consulta tiveram registro no prontuário da pressão arterial aferida pelo menos uma vez, caindo para 64\% em 2017 (Tabela 4). Para aqueles que tiveram o registro da pressão arterial (PA), a proporção de indivíduos controlados foi estável entre 2012 e 2017. A análise dos exames de controle lipídico também não foi satisfatória. Em 2012, aproximadamente 44\% das pessoas com hipertensão tiveram pelo menos um registro dos exames de colesterol total, frações e triglicérides em seus prontuários clínicos. Esse percentual diminuiu para $28 \%$ em 2017 , sendo essa redução estatisticamente significativa a um nível de $5 \%$.

Por fim, realizou-se uma análise pareada ao longo dos seis anos (Gráfico 2), o que permite avaliar a linha de cuidado ao acompanhar o indivíduo em todo o período estudado. Observa-se que tanto para diabetes como para hipertensão, há uma perda expressiva de indivíduos que não realizaram os exames preconizados pelas Linhas Guias (ANDRADE et al., 2017 , 2019). Em relação ao controle lipídico, nota-se que quase $9 \%$ dos indivíduos com DM e 13\% daqueles com HAS não realizaram o exame recomendado (colesterol total, LDL e triglicérides) em nenhum dos anos analisados. A proporção de pessoas que realizaram o exame nos seis anos foi de apenas $7 \%$ para DM e $3 \%$ para HAS. No caso do controle glicêmico, também encontrou-se um percentual elevado de indivíduos que não realizaram o exame em todo o período, especialmente a hemoglobina glicada, cuja proporção alcançou $15 \%$. Apesar das mudanças positivas decorrentes da implementação do LIACC, principalmente para os indivíduos com DM, esses resultados evidenciam ainda uma fragilidade do sistema em prover um cuidado continuado. 0 sistema parece ter aumentado o número de indivíduos com algum exame, mas não está sendo capaz de prover os exames que precisam ser realizados pelo menos uma vez por ano para garantir o cuidado longitudinal. 
TABELA 2

Distribuição dos pacientes com 18 anos e mais com diabetes melittus, segundo resultados dos exames laboratoriais Município de Santo Antônio do Monte, MG - 2012-2017

\begin{tabular}{|c|c|c|c|c|c|c|c|c|c|c|c|c|}
\hline \multirow[t]{2}{*}{ Exames } & \multicolumn{2}{|c|}{2012} & \multicolumn{2}{|c|}{2013} & \multicolumn{2}{|c|}{2014} & \multicolumn{2}{|c|}{2015} & \multicolumn{2}{|c|}{2016} & \multicolumn{2}{|c|}{2017} \\
\hline & $\mathrm{N}$ & $\%$ & $\mathrm{~N}$ & $\%$ & $\mathrm{~N}$ & $\%$ & $\mathrm{~N}$ & $\%$ & $\mathrm{~N}$ & $\%$ & $\mathrm{~N}$ & $\%$ \\
\hline Total & 260 & 100,00 & 260 & 100,00 & 2601 & 100,00 & 260 & 100,00 & 260 & 100,00 & 260 & 100,00 \\
\hline Fez consulta & 234 & 90,00 & 247 & 95,00 & 244 & 93,85 & 242 & 93,08 & 239 & 91,92 & 238 & 91,54 \\
\hline $\begin{array}{l}\text { Registro exame } \\
\text { colesterol total } \\
(\mathrm{mg} / \mathrm{dL})\end{array}$ & 107 & 45,73 & 110 & 44,53 & 120 & 49,18 & 125 & 51,65 & 126 & 52,72 & 122 & 51,26 \\
\hline & 26 & 24,30 & 31 & 28,18 & 45 & 37,50 & 45 & 36,00 & 55 & 43,65 & 48 & 39,34 \\
\hline 60-199 & 33 & 30,84 & 29 & 26,36 & 36 & 30,00 & 32 & 25,60 & 40 & 31,75 & 41 & 33,61 \\
\hline $200-239$ & 24 & 22,43 & 33 & 30,00 & 24 & 20,00 & 31 & 24,80 & 18 & 14,29 & 16 & 13,11 \\
\hline $40-279$ & 12 & 11,21 & 10 & 9,09 & 10 & 8,33 & 10 & 8,00 & 5 & 3,97 & 7 & 5,74 \\
\hline 280 ou m & 8 & 7,48 & 4 & 3,64 & 1 & 0,83 & 3 & 2,40 & 2 & 1,59 & 2 & 1,64 \\
\hline$S$ & 4 & 3,74 & 3 & 2,73 & 4 & 3,33 & 4 & 3,20 & 6 & 4,76 & 8 & 6,56 \\
\hline édia ( \pm & 196,0 & $(49,60)$ & 193,6 & $(51,50)$ & 179,7 & $(44,00)$ & 181 & $(43,70)$ & 170,4 & $(41,7)$ & 175,1 & $(39,20)$ \\
\hline lediana & 189 & & 191 & & 175 & & 172 & & 161,5 & & 169,5 & \\
\hline $\begin{array}{l}\text { Registro } \\
\text { LDL }(\mathrm{mg} / \mathrm{c}\end{array}$ & 104 & 44,44 & 108 & 43,72 & 118 & 48,36 & 125 & 51,65 & 124 & 51,88 & 123 & 51,68 \\
\hline$<100$ & 36 & 34,62 & 43 & 39,81 & 60 & 50,85 & 66 & 52,80 & 80 & 64,52 & 79 & 64,23 \\
\hline$\geq 100$ & 59 & 56,73 & 60 & 55,56 & 52 & 44,07 & 49 & 39,20 & 32 & 25,81 & 33 & 26,83 \\
\hline NS & 9 & 8,65 & 5 & 4,63 & 6 & 5,08 & 10 & 8,00 & 12 & 9,68 & 11 & 8,94 \\
\hline lédia $( \pm D$ & 117,9 & $(44,00)$ & 108,1 & $(39,40)$ & 100,0 & $(34,00)$ & 97,3 & $(38,90)$ & 87,9 & $(36,70)$ & 88,4 & $(33,30)$ \\
\hline Mediana & 116 & & 110 & & 98 & & 90 & & 85 & & 81 & \\
\hline triglicérides & 107 & 45,73 & 111 & 44,94 & 122 & 50,00 & 125 & 51,65 & 127 & 53,14 & 123 & 51,68 \\
\hline 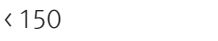 & 46 & 42,99 & 56 & 50, & 58 & 47, & 60 & 48 & 57 & 44,88 & 58 & 47,15 \\
\hline$\geq 150$ & 56 & 52,34 & 52 & 46,85 & 60 & 49,18 & 61 & 48,80 & 64 & 50,39 & 57 & 46,34 \\
\hline NS & 5 & 4,67 & 3 & 2,70 & 4 & 3,28 & 4 & 3,20 & 6 & 4,72 & 8 & 6,50 \\
\hline Média $( \pm D$ & 175,9 & $(93,40)$ & 174,4 & $(107,50)$ & 173,5 & $(93,90)$ & $(175,6)$ & 101,40 & $(174,1)$ & 88,1 & $(176,7)$ & $(114,1)$ \\
\hline Med & 161 & & 147 & & 152 & & 150 & & 153 & & 147 & \\
\hline Registro & 109 & 46,58 & 109 & 44,13 & 122 & 50,00 & 103 & 42,56 & 75 & 31,38 & 97 & 40,76 \\
\hline$<100$ & 14 & 12,84 & 9 & 8,26 & 34 & 27,87 & 21 & 20,39 & 18 & 24,00 & 15 & 15,46 \\
\hline $100-125$ & 31 & 28,44 & 32 & 29,36 & 37 & 30,33 & 21 & 20,39 & 19 & 25,33 & 31 & 31,96 \\
\hline$\geq 126$ & 60 & 55,05 & 66 & 60,55 & 46 & 37,70 & 57 & 55,34 & 31 & 41,33 & 45 & 46,39 \\
\hline NS & 4 & 3,67 & 2 & 1,83 & 5 & 4,10 & 4 & 3,88 & 7 & 9,33 & 6 & 6,19 \\
\hline Média ( & 149,9 & $(59,30)$ & 158,2 & $(62,30)$ & 138,5 & $(65,90)$ & 149,7 & $(63,90)$ & 142,2 & $(66,00)$ & 146,3 & $(59,50)$ \\
\hline Mediana & 132 & & 136 & & 118 & & 128 & & 118,5 & & 125 & \\
\hline $\begin{array}{l}\text { Registro exame de } \\
\text { glicohemoglobina }\end{array}$ & 68 & 29 & 83 & 33 & 103 & 42 & 103 & 6 & 94 & 39,33 & 100 & 42,02 \\
\hline$<7$ & 23 & 33,82 & 31 & 37,35 & 45 & 43,69 & 32 & 31,07 & 40 & 42,55 & 39 & 39,00 \\
\hline $7 \mathrm{as}$ & 22 & 32,35 & 17 & 20,48 & 29 & 28,16 & 31 & 30,10 & 19 & 20,21 & 23 & 23,00 \\
\hline$>9$ & 20 & 29,41 & 34 & 40,96 & 25 & 24,27 & 35 & 33,98 & 29 & 30,85 & 32 & 32,00 \\
\hline NS & 3 & 4,41 & 1 & 1,20 & 4 & 3,88 & 5 & 4,85 & 6 & 6,38 & 6 & 6,00 \\
\hline Média $( \pm \mathrm{DP})$ & 8,7 & $(3,00)$ & 8,9 & $(3,10)$ & 8,1 & $(2,60)$ & 8,7 & $(2,70)$ & 8,6 & $(2,90)$ & 8,7 & $(3,00)$ \\
\hline Mediana & 7,7 & & 8,2 & & 7,2 & & 8,05 & & 7,2 & & 7,3 & \\
\hline $\begin{array}{l}\text { Registro } \\
\text { glicemia ou } \\
\text { glicohemoglobina }\end{array}$ & 112 & 47,86 & 115 & 46,56 & 131 & 53,69 & 120 & 49,59 & 108 & 45,19 & 115 & 48,32 \\
\hline
\end{tabular}

Fonte: SAMONTE $(2013,2015,2018)$. 
TABELA 3

Distribuição dos pacientes com 18 anos e mais com hipertensão arterial sistêmica, segundo resultados dos exames laboratoriais

Município de Santo Antônio do Monte, MG - 2012-2017

\begin{tabular}{|c|c|c|c|c|c|c|c|c|c|c|c|c|}
\hline \multirow{2}{*}{ Exames } & \multicolumn{2}{|c|}{2012} & \multicolumn{2}{|c|}{2013} & \multicolumn{2}{|c|}{2014} & \multicolumn{2}{|c|}{2015} & \multicolumn{2}{|c|}{2016} & \multicolumn{2}{|c|}{2017} \\
\hline & $\mathrm{N}$ & $\%$ & $\mathrm{~N}$ & $\%$ & $\mathrm{~N}$ & $\%$ & $\mathrm{~N}$ & $\%$ & $\mathrm{~N}$ & $\%$ & $\mathrm{~N}$ & $\%$ \\
\hline Total & 295 & 100 & 295 & 100 & 295 & 100 & 295 & 100 & 295 & 100 & 295 & 100 \\
\hline Fez consulta & 238 & 80,7 & 256 & 86,8 & 261 & 88,5 & 248 & 84,1 & 240 & 81,4 & 250 & 84,8 \\
\hline $\begin{array}{l}\text { Registro exame } \\
\text { colesterol total } \\
(\mathrm{mg} / \mathrm{dL})\end{array}$ & 105 & 44,1 & 78 & 30,5 & 96 & 36,8 & 94 & 37,9 & 90 & 37,5 & 69 & 27,6 \\
\hline$<160$ & 15 & 14,3 & 14 & 18,0 & 9 & 9,4 & 15 & 16,0 & 20 & 22,2 & 16 & 23,2 \\
\hline 160-199 & 31 & 29,5 & 28 & 35,9 & 39 & 40,6 & 31 & 33,0 & 32 & 35,6 & 22 & 31,9 \\
\hline $200-239$ & 40 & 38,1 & 23 & 29,5 & 31 & 32,3 & 25 & 26,6 & 23 & 25,6 & 18 & 26,1 \\
\hline $240-279$ & 9 & 8,6 & 8 & 10,3 & 10 & 10,4 & 9 & 9,6 & 6 & 6,7 & 6 & 8,7 \\
\hline 280 ou mais & 7 & 6,7 & 4 & 5,1 & 1 & 1,0 & 7 & 7,5 & 4 & 4,4 & 0 & 0,0 \\
\hline NS & 3 & 2,9 & 1 & 1,3 & 6 & 6,3 & 7 & 7,5 & 5 & 5,6 & 7 & 10,1 \\
\hline Média ( $\pm D P)$ & 205,6 & $(46,1)$ & 201,4 & $(45,0)$ & 198,0 & $(35,8)$ & 200,5 & $(52,2)$ & 193,0 & $(42,2)$ & 187,5 & $(38,4)$ \\
\hline Mediana & 201,0 & & 196,6 & & 193,5 & & 198,0 & & 184,0 & & 186,0 & \\
\hline $\begin{array}{l}\text { Registro exame LDL } \\
(\mathrm{mg} / \mathrm{dL})\end{array}$ & 105 & 44,1 & 76 & 29,7 & 95 & 36,4 & 93 & 37,5 & 88 & 36,7 & 68 & 27,2 \\
\hline$<100$ & 18 & 17,1 & 26 & 34,2 & 25 & 26,3 & 32 & 34,4 & 43 & 48,9 & 29 & 42,7 \\
\hline$\geq 100$ & 82 & 78,1 & 49 & 64,5 & 67 & 70,5 & 53 & 57,0 & 40 & 45,5 & 30 & 44,1 \\
\hline NS & 5 & 4,8 & 1 & 1,3 & 3 & 3,2 & 8 & 8,6 & 5 & 5,7 & 9 & 13,2 \\
\hline Média ( $\pm \mathrm{DP})$ & 132,0 & $(38,1)$ & 115,3 & $(37,3)$ & 117,0 & $(32,4)$ & 115,8 & $(42,6)$ & 102,4 & $(34,4)$ & 102,8 & $(36,5)$ \\
\hline Mediana & 126,0 & & 108,0 & & 115,0 & & 110,0 & & 96,0 & & 101,0 & \\
\hline $\begin{array}{l}\text { Registro exame } \\
\text { triglicérides }(\mathrm{mg} / \mathrm{dL})\end{array}$ & 104 & 43,7 & 80 & 31,3 & 94 & 36,0 & 93 & 37,5 & 88 & 36,7 & 70 & 28,0 \\
\hline$<150$ & 67 & 64,4 & 50 & 62,5 & 58 & 61,7 & 41 & 44,1 & 50 & 56,8 & 32 & 45,7 \\
\hline$\geq 150$ & 34 & 32,7 & 30 & 37,5 & 33 & 35,1 & 41 & 44,1 & 34 & 38,6 & 31 & 44,3 \\
\hline NS & 3 & 2,9 & 0 & 0,0 & 3 & 3,2 & 11 & 11,8 & 4 & 4,6 & 7 & 10,0 \\
\hline Média ( $\pm \mathrm{DP})$ & 141,1 & $(88,1)$ & 148,9 & $(68,9)$ & 154,1 & $(106,3)$ & 172,3 & $(81,8)$ & 154,9 & $(81,4)$ & 152,2 & $(69,5)$ \\
\hline Mediana & 127,0 & & 137,0 & & 117,0 & & 150,5 & & 129,0 & & 149,0 & \\
\hline $\begin{array}{l}\text { Teve pressão arterial } \\
\text { medida }(\mathrm{mmHg})\end{array}$ & 191 & 80,3 & 170 & 66,4 & 151 & 57,9 & 140 & 56,5 & 160 & 66,7 & 160 & 64,0 \\
\hline$<90$ & 143 & 74,9 & 123 & 72,4 & 108 & 71,5 & 109 & 77,9 & 128 & 80,0 & 122 & 76,3 \\
\hline$\geq 90$ & 48 & 25,1 & 47 & 27,7 & 43 & 28,5 & 30 & 21,4 & 32 & 20,0 & 38 & 23,8 \\
\hline NS & 0 & 0,0 & 0 & 0,0 & 0 & 0,0 & 1 & 0,7 & 0 & 0,0 & 0 & 0,0 \\
\hline Média ( $\pm D P)$ & 80,5 & $(13,2)$ & 80,9 & $(11,1)$ & 80,8 & $(12,8)$ & 79,1 & $(10,6)$ & 79,5 & $(10,9)$ & 80,1 & $(11,5)$ \\
\hline Mediana & 80,0 & & 80,0 & & 80,0 & & 80,0 & & 80,0 & & 80,0 & \\
\hline
\end{tabular}

Fonte: SAMONTE $(2013,2015,2018)$. 


\section{GRÁFICO 2}

Frequência acumulada de exames laboratoriais realizados por pacientes nos anos avaliados Município de Santo Antônio do Monte, MG - 2012/2017

LDL realizado em

150

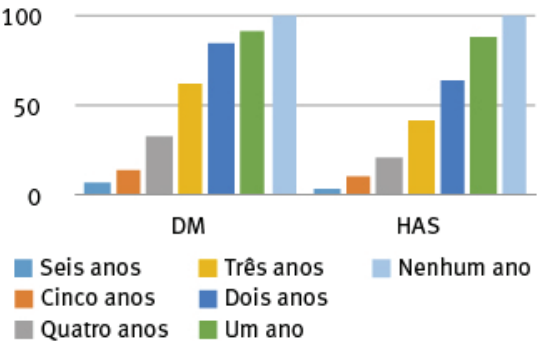

Colesterol realizado em

150

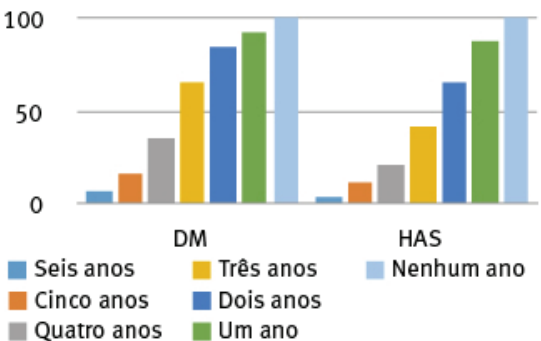

Controle glicêmico em pacientes com DM realizado em

150

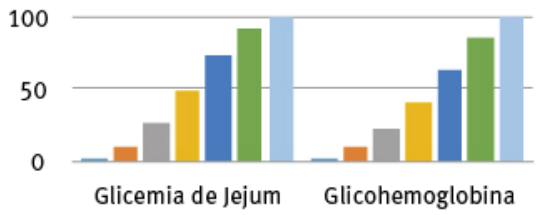

Seis anos $\quad$ Três anos Nenhum ano

Cinco anos Dois anos

- Quatro anos Um ano

Fonte: SAMONTE $(2013,2015,2018)$.
Consultas realizadas em

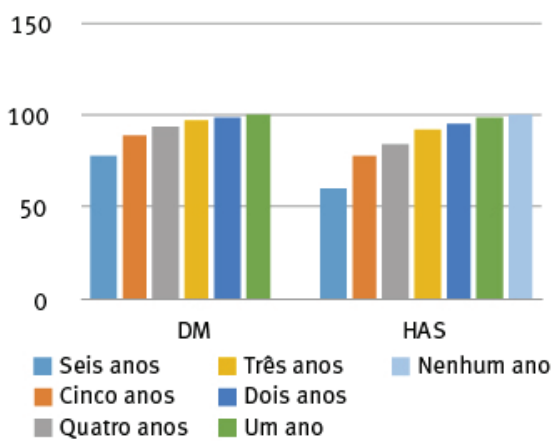

Triglicérides realizado em

150

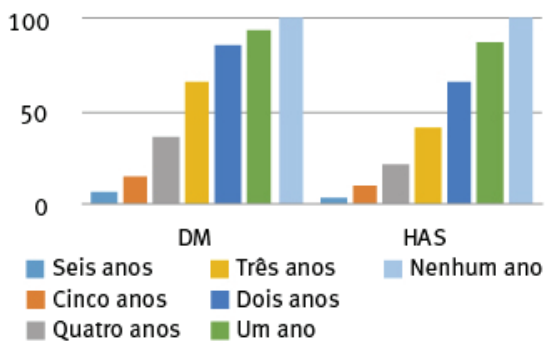

Pressão aferida em pacientes com HAS

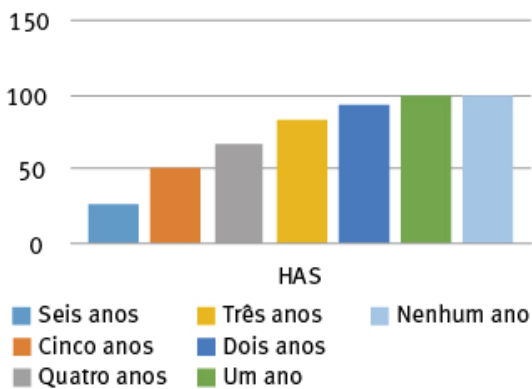

\section{Discussão}

A despeito da elevada carga de morbimortalidade associada ao diabetes mellitus e à hipertensão arterial sistêmica no Brasil e no mundo, poucos indivíduos têm recebido o 
tratamento contínuo e integralizado (CROWLEY, 2013; HUQUE et al., 2018). A atual crise dos sistemas de saúde encontra-se no descompasso entre a necessidade da adoção de estratégias contínuas e integradas e o modelo vigente que prioriza o tratamento das condições agudas e agudizações das condições crônicas por meio de um modelo de cuidado fragmentado (MENDES, 2018). Nessa perspectiva, a estruturação das redes de atenção à saúde com foco na atenção às condições crônicas emerge como um dos principais planos de ação para lidar com essa questão (MENDES et al., 2019).

Segundo Mendes (2018), esse não é um problema exclusivo do Brasil, ocorrendo em todo o mundo e até mesmo nos países desenvolvidos. Ainda de acordo com o autor, um levantamento conduzido no Reino Unido demonstrou que, entre 100 pessoas com doenças crônicas, apenas metade delas está diagnosticada. E, entre estas últimas, somente metade encontra-se estabilizada e inscrita em programas de prevenção e promoção da saúde para o controle das condições crônicas. Os resultados são semelhantes para os países em desenvolvimento. Em Bangladesh, um estudo randomizado controlado para controle da hipertensão demonstrou que somente metade dos indivíduos recebeu o acompanhamento de forma adequada. Entre as principais causas apontadas estão os diagnósticos incompletos e a não provisão de consultas de acompanhamento (HUQUE et al., 2018). No Brasil, as evidências apontam para a mesma direção. Um estudo com pacientes de 22 centros clínicos brasileiros mostrou que apenas $10 \%$ e $25 \%$ dos pacientes com diabetes mellitus tipo I e II, respectivamente, estavam com os níveis de açúcar no sangue controlados. Quase metade dos pacientes apresentou sinais de doença renal e neuropatias e $16 \%$ tinham insuficiência renal (DOMINGUEZ, 2007; MENDES, 2019).

o Chronic Care Model, desenvolvido nos Estado Unidos, foi o principal modelo adotado pelos países desenvolvidos e por alguns países em desenvolvimento para o manejo das condições crônicas (MENDES, 2018). No Brasil, ao final de 2010, foram estabelecidas as diretrizes para a organização das redes de atenção à saúde no âmbito do Sistema Único de Saúde (BRASIL, 2014). 0 modelo implementado no SUS combina elementos do Chronic Care Model com outros do modelo da pirâmide de risco, que se baseia na estratificação do risco das pessoas portadoras de condições crônicas, e do modelo da determinação social, ancorado na compreensão das características sociais específicas da população atendida (MENDES, 2018). Desde então, programas e intervenções assistenciais com vista à promoção da saúde por meio do manejo adequado das condições crônicas vêm sendo adotados em diversas localidades do país, apresentando-se, no geral, como casos bem-sucedidos (MENDES et al., 2019).

Atualmente, dez estados brasileiros participam do projeto de Planificação da Atenção Primária à Saúde (Paps), proposta desenvolvida pelo Conselho Nacional de Secretários de Saúde (CONASS, 2018), visando a integração da APS com a atenção ambulatorial especializada a partir de mudanças dos processos de trabalho. As primeiras experiências de Paps se deram com a implementação doLIACC em Curitiba, Santo Antônio do Monte e Tauá, locais 
onde esse modelo pôde ser testado. Com base nessas experiências, foi possível expandir o projeto para outros locais (MENDES, 2016). Em Curitiba, por exemplo, o LIACC resultou em melhoras dos processos, sobretudo os relacionados à articulação com a comunidade e ao suporte à decisão, baseados no desenvolvimento do cuidado compartilhado e autocuidado apoiado (MOYSES et al., 2012). No Distrito Federal, a implantação da Paps acarretou um aumento da cobertura populacional pelas equipes da ESF de $34 \%$, em 2017, para $69 \%$, em 2018. Foram observadas também melhoras na linha de cuidado de diabetes e hipertensão entre 2016 e 2018 (EVANGELISTA et al., 2019; TASCA et al., 2019).

A experiência particular de Santo Antônio do Monte é sem dúvida uma das mais notáveis (MENDES, 2019). A contribuição inédita deste estudo está na avaliação da linha de cuidado de uma coorte de indivíduos com diabetes e hipertensão, com base nos registros clínicos dos prontuários médicos dos usuários do SUS no município. Nessa lógica, o presente estudo se diferencia dos demais realizados para o Brasil, os quais concentram atenção nos resultados acerca da percepção profissional sobre a capacidade institucional e da aplicabilidade do modelo de atenção às condições crônicas (COSTA et al., 2018; TASCA et al., 2019; EVANGELISTA et al., 2019; RIBEIRO et al., 2019).

No caso de SAMONTE, os resultados obtidos apontam para o LIACC como uma intervenção importante para a introdução dos processos estipulados pelas RAS-CC. Observa-se um aumento na classificação de risco familiar e na implementação da estratificação de risco individual. Houve um incremento significativo tanto na proporção de consultas realizadas quanto na proporção de exames de glicohemoglobina e perfil lipídico registrados nos prontuários de indivíduos com diabetes. No entanto, para aqueles com hipertensão, ainda que haja um crescimento na proporção de consultas, os resultados relativos aos exames preconizados pelas Linhas Guias não são favoráveis ao LIACC, uma vez que ocorreu redução da cobertura desses exames no período analisado.

Em suma, pode-se dizer que o LIACC foi efetivo na universalização do cuidado, a partir da ampliação do acesso à população aos serviços de saúde. Contudo, permanecem muitos desafios, sobretudo no acompanhamento longitudinal e na realização dos exames de diagnósticos entre indivíduos crônicos. Como exposto anteriormente, essa realidade não se restringe a SAMONTE, sendo observada também em outras localidades no Brasil e no mundo.

A incorporação dos protocolos preconizados requer mudanças no processo de trabalho. A rotatividade de médicos, os baixos incentivos salariais para outros profissionais da saúde e mudanças políticas são fatores difíceis de avaliar em curto espaço de tempo, mas que refletem diretamente na qualidade da assistência oferecida. Dentre outros gargalos apontados pela literatura, estão o desfalque no quadro de funcionários para a participação dos treinamentos, a dificuldade encontrada nos processos de referenciamento para especialistas nos casos mais graves, um cartão único para o acompanhamento dos usuários e ainda a falta de medicamentos nos postos de saúde (HUQUE et al., 2018). 


\section{Limitações}

Este estudo apresenta algumas limitações. A primeira refere-se ao curto período de tempo para avaliação da implementação da linha de cuidado no âmbito do LIACC. A intervenção resultou em mudanças em alguns processos de trabalho da RAS que requerem tempo para produzir resultados clínicos (MENDES, 2012). Um segundo aspecto diz respeito à fonte de dados. As informações dos registros clínicos, principalmente de exames, podem não refletir a realidade devido à presença de sub-registro. Por fim, cabe mencionar que a escolha de SAMONTE não foi aleatória. A presença do Centro Hiperdia/Viva Vida, considerado um modelo de atendimento adequado a indivíduos de alto risco com diabetes e hipertensão, foi o principal critério para sua escolha como município-piloto (BRASIL, 2013). A adoção desse critério foi importante, uma vez que a ausência de uma atenção secundária organizada seria um gargalo para o MACC. SAMONTE é um município polo da microrregião de saúde Divinópolis e o Centro Hiperdia/Viva Vida realiza atendimentos a indivíduos com hipertensão e diabetes dos municípios da região desde 2011 (BRASIL, 2013).

\section{Conclusão}

A linha de cuidado ofertada para os indivíduos com diabetes em SAMONTE ainda não atingiu seu ápice de qualidade, embora mudanças positivas tenham sido observadas com a implantação do LIACC. A alta cobertura da APS no município e o reconhecimento dos indivíduos nas UBS foram pontos positivos, porém, os princípios de longitudinalidade no acompanhamento permanecem insuficientes. Uma análise por seis anos a partir de prontuários, pouco vista na literatura, permite afirmar que a maioria dos indivíduos, principalmente com diabetes acompanhados pela APS, apresentou bom controle metabólico. Desse modo, o LIACC promoveu uma melhora no processo e manejo das condições crônicas, sendo uma evidência positiva para a implementação das RAS-CC no SUS.

\section{Referências}

ANDRADE, M. V. et al. Avaliação do laboratório de inovações no cuidado das condições crônicas na atenção primária à saúde de Santo Antônio do Monte. Belo Horizonte: Centro de Desenvolvimento e Planejamento Regional (Cedeplar), Universidade Federal de Minas Gerais (UFMG), 2017. (Texto para Discussão, n. 552).

ANDRADE, M. V. et al. Challenges and lessons from a primary care intervention in a Brazilian municipality. Revista de Saúde Pública (online), v. 53, p. 45, 2019.

BAHIA, L. R. et al. The costs of type 2 diabetes mellitus outpatient care in the Brazilian public health system. Value in Health, v. 14, n. 5, p. S137-S140, 2011.

BELTRÁN-SÁNCHEZ, H. et al. Links between socio-economic circumstances and changes in smoking behavior in the Mexican population: 2002-2010. Journal of Cross-Cultural Gerontology, v. 28, n. 3, p. 339-358, 2013. 
BRASIL. Ministério da Saúde. Secretaria de Vigilância em Saúde. Departamento de Análise de Situação de Saúde. Plano de Ações Estratégicas para o Enfrentamento das Doenças Crônicas Não Transmissíveis (DCNT) no Brasil 2011-2022. Brasília: Ministério da Saúde, 2011.

BRASIL. Ministério da Saúde. Portaria no 4279, de 30 de dezembro de 2010. Estabelece diretrizes para a organização da Rede de Atenção à Saúde no âmbito do Sistema Único de Saúde (SUS). Brasília, 2010.

BRASIL. Ministério da Saúde. Diretrizes para o cuidado das pessoas com doenças crônicas nas redes de atenção à saúde e nas linhas de cuidado prioritárias. Brasília: Secretaria de Atenção à Saúde, Departamento de Atenção Básica, 2013.

CANUDAS-ROMO, V.; GARCÍA-GUERRERO, V. M.; ECHARRI-CÁNOVAS, C. J. The stagnation of the Mexican male life expectancy in the first decade of the 21st century: the impact of homicides and diabetes mellitus. Journal of Epidemiology \& Community Health, v. 69, n. 1, p. 28-34, 2015.

COELI, C. M. et al. Mortalidade em idosos por diabetes mellitus como causa básica e associada. Revista de Saúde Pública, v. 36, p. 135-140, 2002.

CONASS - Conselho Nacional de Secretários de Saúde. Planificação da Atenção à Saúde. 2018. Disponível em: 〈https://www.conass.org.br/planificacao-da-atencao-saude/〉. Acesso em: 27 out. 2019.

CONDE, W. L.; MONTEIRO, C. A. Nutrition transition and double burden of undernutrition and excess of weight in Brazil. The American Journal of Clinical Nutrition, v. 100, n. 6, p. 1617S-1622S, 2014.

COOPER, J. G. et al. Quality of care for patients with type 2 diabetes in primary care in Norway is improving: results of cross-sectional surveys of 33 general practices in 1995 and 2005. Diabetes Care, v. 32, n. 1, p. 81-83, 2009.

COSTA, A. F. et al. Carga do diabetes mellitus tipo 2 no Brasil. Cadernos de Saúde Pública, v. 33, p. e00197915, 2017.

COSTA, K. C.; CAZOLA, L. H. O.; TAMAKI, E. M. Assessment of Chronic Illness Care (ACIC): avaliação da aplicabilidade e resultados. Saúde em Debate, v. 40, p. 106-117, 2016.

CROWLEY, M. J. et al. Tailored case management for diabetes and hypertension (TEACH-DM) in a community population: study design and baseline sample characteristics. Contemporary Clinical Trials, v. 36, n. 1, p. 298-306, 2013.

DE ALMEIDA-PITITTO, B. et al. Type 2 diabetes in Brazil: epidemiology and management. Diabetes, Metabolic Syndrome and Obesity: Targets and Therapy, v. 8, p. 17, 2015.

DE CASTRO MOREIRA, L. M. et al. Políticas públicas voltadas para a redução da mortalidade infantil: uma história de desafios. Revista Médica de Minas Gerais, v. 22, supl. 7, p. S48-S55, 2012.

EVANGELISTA, M. J. O. et al. O planejamento e a construção das redes de atenção à saúde no DF, Brasil. Ciência \& Saúde Coletiva, v. 24, n. 6, p. 2115-2124, 2019.

FERTONANI, H. P. et al. Modelo assistencial em saúde: conceitos e desafios para a atenção básica brasileira. Ciência \& Saúde Coletiva, v. 20, n. 6, p. 1869-1878, 2015.

FONTBONNE, A. et al. Risk factor control in hypertensive and diabetic subjects attended by the Family Health Strategy in the State of Pernambuco, Brazil: the SERVIDIAH study. Cadernos de Saúde Pública, v. 29, n. 6, p. 1195-1204, 2013.

GILMER, T. P. et al. Cost-effectiveness of diabetes case management for low income populations. Health Services Research, v. 42, n. 5, p. 1943-1959, 2007. 
HOERGER, T. J. et al. Cost-effectiveness of intensive glycemic control, intensified hypertension control, and serum cholesterol level reduction for type 2 diabetes. Jama-Journal of the American Medical Association, v. 287, n. 19, p. 2542-2551, 2002.

HORIUCHI, S. Epidemiological transitions in human history. Health and mortality: issues of global concern. Proceedings of the Symposium on Health and Mortality, Brussels, 19-22 November 1997. New York: United Nations, Department of Economic and Social Affairs, Population Division, 1999. p. 54-71.

HUQUE, R. et al. Integrating a diabetes and hypertension case management package within primary health care: a mixed methods feasibility study in Bangladesh. BMC Health Services Research, v. 18, n. 1, p. 811, 2018.

KLAFKE, A. et al. Mortalidade por complicações agudas do diabetes melito no Brasil, 2006-2010. Epidemiologia e Serviços de Saúde, v. 23, n. 3, p. 455-462, 2014.

KREIN, S. L. et al. Case management for patients with poorly controlled diabetes: a randomized trial. The American Journal of Medicine, v. 116, n. 11, p. 732-739, 2004.

LOBO, L. A. C. et al. Tendência temporal da prevalência de hipertensão arterial sistêmica no Brasil. Cadernos de Saúde Pública, v. 33, p. e00035316, 2017.

LUNA, R. L.; LUNA, L. C. Estaria a pressão diastólica perdendo sua utilidade na clínica? Arquivos Brasileiros de Cardiologia, v. 89, n. 3, p. e19-e21, 2007.

LV, Y. Y. et al. Evaluation on the case management program of hypertension in Zhongguancun community, Beijing. Zhonghua liu xing bing xue za zhi = Zhonghua liuxingbingxue zazhi, v. 26, n. 6, p. 400-403, 2005.

MALFATTI, C. R. M.; ASSUNÇÃO, A. N. Hipertensão arterial e diabetes na Estratégia de Saúde da Família: uma análise da frequência de acompanhamento pelas equipes de Saúde da Família. Ciência \& Saúde Coletiva, v. 16, supl. 1, p. 1383-1388, 2011.

MALTA, D. C.; MERHY, E. E. O percurso da linha do cuidado sob a perspectiva das doenças crônicas não transmissíveis. Interface - Comunicação, Saúde, Educação, v. 14, n. 34, p. 593-606, 2010.

MALTA, D. C. et al. Fatores de risco relacionados à carga global de doença do Brasil e Unidades Federadas, 2015. Revista Brasileira de Epidemiologia, v. 20, supl. 1, p. 217-232, 2017.

MEIRELES, L. A. et al. Linha-guia de hipertensão arterial sistêmica, diabetes mellitus e doença renal crônica. Belo Horizonte: Secretaria de Estado de Saúde de Minas Gerais: Autêntica Editora, 2013.

MENDES, E. V. As redes de atenção à saúde. Brasília: Organização Pan-Americana da Saúde, 2011.

MENDES, E. V. 0 cuidado das condições crônicas na atenção primária à saúde: 0 imperativo da consolidação da estratégia da saúde da família. Brasília: Organização Pan-Americana de Saúde Pública, Organização Mundial da Saúde, Conselho Nacional de Secretários de Saúde, 2012.

MENDES, E. V. Entrevista. Consensus - Revista do Conselho Nacional de Secretários de Saúde, ano VI, n. 20, p. 6-17, jul./set. 2016. Disponível em: 〈https://www.conass.org.br/biblioteca/pdf/ revistaconsensus_20.pdf〉. Acesso em: 27 out. 2019.

MENDES, E. V. The care for chronic conditions in primary health care. Revista Brasileira em Promoção da Saúde, v. 31, n. 2, p. 1-3, 2018.

MENDES, E. V. et al. Integrated care in the unified health system of Brazil: the laboratory for innovation in chronic conditions in Santo Antônio do Monte. International Journal of Healthcare Management, v. 12, n. 2, p. 116-122, 2019. 
MOYSÉS, S. T. et al. Laboratório de inovações no cuidado das condições crônicas na APS: a implantação do Modelo de Atenção às Condições Crônicas na UBS Alvorada em Curitiba. Paraná, 2013.

NORRIS, S. L. et al. The effectiveness of disease and case management for people with diabetes: a systematic review. American Journal of Preventive Medicine, v. 22, n. 4, p. 15-38, 2002.

POBLETE, F. et al. Efectividad de un modelo de manejo de pacientes con hipertensión y diabetes tipo II en atención primaria. Revista Médica de Chile, v. 146, n. 11, p. 1269-1277, 2018.

POPKIN, B. M.; ADAIR, L. S.; NG, S. W. Global nutrition transition and the pandemic of obesity in developing countries. Nutrition Reviews, v. 70, n. 1, p. 3-21, 2012.

PRUITT, S. Innovative care for chronic conditions: building blocks for action: global report. World Health Organization, 2002.

ROSA, R. S. et al. Internações por diabetes mellitus como diagnóstico principal na rede pública do Brasil, 1999-2001. Revista Brasileira de Epidemiologia, v. 10, n. 4, p. 465-478, 2007.

RIBEIRO, M. A. et al. Organização do cuidado às condições crônicas na atenção primária à saúde de Sobral-CE: avaliação de processo na perspectiva de gestores. APS em Revista, v. 1, n. 1, p. 29-38, 2019.

SAFAR, M. Therapeutic trials and large arteries in hypertension. American Heart Journal, v. 115, n. 3, p. 702-710, 1988.

SARTORELLI, D. S.; FRANCO, L. J. Tendências do diabetes mellitus no Brasil: o papel da transição nutricional. Cadernos de Saúde Pública, v. 19, supl. 1, p. S29-S36, 2003.

SILVEIRA, J. A. A. et al. Características da assistência à saúde a pessoas com diabetes mellitus acompanhadas na Unidade de Saúde da Família Pedregal II em Cuiabá, MT: reflexões para a equipe de saúde. 0 Mundo da Saúde, v. 34, n. 1, p. 43-49, 2010.

SCHMIDT, M. I. et al. Chronic non-communicable diseases in Brazil: burden and current challenges. The Lancet, v. 377, n. 9781, p. 1949-1961, 2011.

TASCA, R. et al. Laboratórios de inovação em saúde: por uma Atenção Primária à Saúde forte no Distrito Federal, Brasil. Ciência \& Saúde Coletiva, v. 24, n. 6, p. 2021-2030, 2019.

UK PROSPECTIVE DIABETES STUDY GROUP et al. Tight blood pressure control and risk of macrovascular and microvascular complications in type 2 diabetes: UKPDS 38. The BMJ: British Medical Journal, v. 317, n. 7160, p. 703, 1998.

VENANCIO, S. I.; ROSA, T. E. C.; BERSUSA, A. A. S. Atenção integral à hipertensão arterial e diabetes mellitus: implementação da linha de cuidado em uma Região de Saúde do estado de São Paulo, Brasil. Physis: Revista de Saúde Coletiva, v. 26, n. 1, p. 113-135, 2016.

VIEIRA, P. L. et al. Valores de LDL-colesterol estimados pela equação de Friedewald são afetados pelo controle do diabetes. International Journal of Cardiovascular Sciences, v. 29, n. 5 , p. f: 348-l: 354, 2016.

\section{Sobre as autoras}

Mônica Viegas Andrade é economista, doutora em economia pela Escola de Pós-Graduação em Economia Fundação Getúlio Vargas. Professora associada da Universidade Federal de Minas Gerais (UFMG). Departamento de Economia. Centro de Desenvolvimento e Planejamento Regional (Cedeplar). 
Kenya Noronha é economista, doutora em economia pelo Centro de Desenvolvimento e Planejamento Regional (Cedeplar/UFMG). Professora associada da Universidade Federal de Minas Gerais. Departamento de Economia. Centro de Desenvolvimento e Planejamento Regional (Cedeplar).

Cláudia Di Lorenzo Oliveira é médica, doutora em saúde pública pela Universidade Federal de Minas Gerais (UFMG). Professora adjunta da Universidade Federal de São João del-Rei, campus Centro-Oeste, Departamento de Saúde Coletiva.

Clareci Silva Cardoso é psicóloga, doutora em saúde pública pela Universidade Federal de Minas Gerais (UFMG). Professora associada da Universidade Federal de São João del-Rei, campus Centro-Oeste, Departamento de Saúde Coletiva.

Júlia Almeida Calazans é economista, doutoranda e mestre em demografia pelo Centro de Desenvolvimento e Planejamento Regional (Cedeplar), Universidade Federal de Minas Gerais (UFMG).

Nayara Abreu Julião é economista, doutoranda em economia no Centro de Desenvolvimento e Planejamento Regional (Cedeplar), Universidade Federal de Minas Gerais (UFMG) e mestre em economia pela Universidade Federal do Paraná (UFPR).

Aline de Souza é economista, mestranda em economia no Centro de Desenvolvimento e Planejamento Regional (Cedeplar), Universidade Federal de Minas Gerais (UFMG).

Patrícia Aparecida Tavares é fisioterapeuta, doutoranda em ciências da saúde na Universidade Federal de São João del-Rei (UFSJ). Professora da Universidade Estadual de Minas Gerais (UEMG).

\title{
Endereço para correspondência
}

\author{
Mônica Viegas Andrade \\ Universidade Federal de Minas Gerais, Faculdade de Ciências Econômicas, Centro de \\ Desenvolvimento e Planejamento Regional \\ Av. Antônio Carlos, 6627, Pampulha \\ 31270-901 - Belo Horizonte-MG, Brasil \\ Kenya Valeria Micaela de Souza Noronha \\ Universidade Federal de Minas Gerais, Faculdade de Ciências Econômicas, Centro de \\ Desenvolvimento e Planejamento Regional \\ Av. Antônio Carlos, 6627, Pampulha \\ 31270-901 - Belo Horizonte-MG, Brasil \\ Cláudia Di Lorenzo Oliveira \\ Universidade Federal de São João Del-Rei, campus CCO/Dona Lindu \\ Av. Sebastião Gonçalves Coelho, 400, bloco A, sala 208 Chanadour \\ 35501296 - Divinópolis-MG, Brasil \\ Clareci Silva Cardoso \\ Universidade Federal de São João Del-Rei, campus CCO/Dona Lindu \\ Av. Sebastião Gonçalves Coelho, 400, bloco A, sala 208 Chanadour \\ 35501296 - Divinópolis-MG, Brasil \\ Júlia Almeida Calazans \\ Universidade Federal de Minas Gerais, Faculdade de Ciências Econômicas, Centro de \\ Desenvolvimento e Planejamento Regional \\ Av. Antônio Carlos, 6627, Pampulha \\ 31270-901 - Belo Horizonte-MG, Brasil
}




\author{
Nayara Abreu Julião \\ Universidade Federal de Minas Gerais, Faculdade de Ciências Econômicas, Centro de \\ Desenvolvimento e Planejamento Regional \\ Av. Antônio Carlos, 6627, Pampulha \\ 31270-901 - Belo Horizonte-MG, Brasil \\ Aline Souza \\ Universidade Federal de Minas Gerais, Faculdade de Ciências Econômicas, Centro de \\ Desenvolvimento e Planejamento Regional \\ Av. Antônio Carlos, 6627, Pampulha \\ 31270-901 - Belo Horizonte-MG, Brasil \\ Patrícia Aparecida Tavares \\ Universidade Federal de São João Del-Rei, campus CCO/Dona Lindu \\ Av. Sebastião Gonçalves Coelho, 400, bloco A, sala 208 Chanadour \\ 35501296 - Divinópolis-MG, Brasil
}

\title{
Abstract \\ Analysis of the care of patients with Diabetes Mellitus and Hypertension: the experience of a small municipality in Brazil
}

This article evaluates the care of a cohort of 260 individuals with diabetes and 295 individuals with hypertension before, during and after the implementation of the Innovative Care Laboratory for Chronic Conditions (LIACC). This intervention sought to strengthen Primary Health Care by implementing the Chronic Care Model in Santo Antônio do Monte, Minas Gerais, Brazil, between 2013 and 2014. This is a longitudinal observational study that uses clinical and laboratory information from medical records of patients with these two chronic conditions between 2012 and 2017. The outcomes evaluated were based on the guidelines of the Department of Health of Minas Gerais State. The results show the LIACC associated with the universalization of primary care macro-processes such as registration and classification of family risk. For patients with diabetes, there was an improvement in several markers in the period, such as increased consultation (from $90 \%$ in 2012 to $92 \%$ in 2017) and a decrease in patients with examinations outside the normal range. For individuals with hypertension, although there is a marked increase in medical appointments (from $80 \%$ in 2012 to $84 \%$ in 2017), clinical improvements were less evident. It can be concluded that LIACC is a promising intervention to improve the management of patients with chronic diseases in Primary Health Care.

Keywords: Chronic conditions. Primary health care. Cohort studies.

\section{Resumen}

Análisis de la línea de cuidado para pacientes con diabetes mellitus e hipertensión arterial: la experiencia de un municipio pequeño en Brasil

El artículo evalúa la línea de cuidado de una cohorte de 260 individuos con diabetes mellitus y de 295 individuos con hipertensión arterial sistémica antes, durante y después de la implantación del Laboratorio de Innovación en Atención a las Condiciones Crónicas (LIACC). Esta intervención buscó fortalecer la Atención Primaria en Salud implantando el Modelo de Atención a las Condiciones Crónicas en el municipio de Santo Antônio do Monte, Minas Gerais, Brasil, entre 
2013 y 2014. Se trata de un estudio observacional longitudinal con informaciones clínicas y de laboratorio de registros médicos de los pacientes con las dos condiciones crónicas entre 2012 y 2017. Los desenlaces evaluados se basaron en las líneas guía de la Secretaría de Salud del estado de Minas Gerais. Los resultados evidencian el LIACC asociado con la universalización de los macroprocesos de atención primaria como el registro y la clasificación del riesgo familiar. Para los pacientes con diabetes se observó una mejora en muchos marcadores en el período, como el aumento de las consultas (de $90 \%$ en 2012 a 92 \% en 2017) y la disminución de los pacientes con exámenes fuera de los rangos normales. Aunque para las personas con hipertensión se ha observado un notable aumento en la concreción de las consultas (de 80 \% en 2012 a $84 \%$ en 2017), las mejorías clínicas fueron menos pronunciadas. Se concluye que el LIACC es una prometedora intervención para la mejora del manejo de pacientes con enfermedades crónicas en la atención primaria.

Palabras clave: Condiciones crónicas. Atención primaria en salud. Estudios de cohorte.

Recebido para publicação em 20/09/2019

Aceito para publicação em 29/10/2019 